\title{
Uniform Lorentz norm estimates for convolution operators
}

\section{Youngwoo Choi*}

\section{*Correspondence:}

youngwoo@ajou.ac.kr

Department of Mathematics, Ajou University, 206 Worldcup-ro, Suwon, 443-749, South Korea

\begin{abstract}
Uniform endpoint Lorentz norm improving estimates for convolution operators with affine arclength measure supported on simple plane curves are established. The estimates hold for a wide class of simple curves, and the condition is stated in terms of averages of the square of the affine arclength weight, extending previously known results.
\end{abstract}

MSC: Primary 44A35; secondary 42B35

Keywords: affine arclength; convolution; Lorentz space

\section{Introduction}

Let $\phi:(a, b) \rightarrow \mathbb{R}$ be a $C^{2}$ function such that $\phi^{\prime \prime}(t) \geq 0$ for all $t \in(a, b)$. In this paper, we consider the convolution operator $\mathcal{T}$ given by

$$
\mathcal{T} f\left(x_{1}, x_{2}\right)=\int_{a}^{b} f\left(x_{1}-t, x_{2}-\phi(t)\right) \omega(t) d t
$$

for $f \in C_{0}^{\infty}\left(\mathbb{R}^{2}\right)$. Here and in what follows, we denote $\omega(t):=\left(\phi^{\prime \prime}(t)\right)^{1 / 3}$. Curves of the form $(t, \phi(t))$ are said to be simple according to Drury and Marshall [1]. The measure $\omega(t) d t$ supported on the curve $(t, \phi(t))$ is known as the affine arclength measure, which is based on the affine arclength parameter as in [2], and was introduced by Drury and Marshall [1] in dealing with the Fourier restriction problem related to curves, and later by Drury [3] in studying convolution operators with measures supported on curves. We refer interested readers to [2-4] for the relevance of affine geometry in this subject. One big benefit of using the affine arclength measure in place of the Euclidean arclength measure $\sqrt{1+\phi^{\prime}(t)^{2}} d t$ has been its effect of mitigating degeneracies and it is believed that various uniform sharp estimates hold for a wide class of curves.

As is well known, the typeset $\mathcal{S}=\left\{\left(p^{-1}, q^{-1}\right): \mathcal{T}\right.$ is bounded from $L^{p}\left(\mathbb{R}^{2}\right)$ to $\left.L^{q}\left(\mathbb{R}^{2}\right)\right\}$ of $\mathcal{T}$ is contained in the convex hull of $\{(0,0),(1,1),(2 / 3,1 / 3)\}$ and uniform estimates in $a, b$, and $\phi$ are expected only for $(1 / p, 1 / q)=(2 / 3,1 / 3)$. Many conditions to guarantee optimal uniform $L^{3 / 2}-L^{3}$ estimates have been known so far. See [3, 5-12] for example. Among other things, the author proved the following.

Theorem 1.1 (Choi [12]) Let $J$ be an open interval in $\mathbb{R}$, and $\phi: J \rightarrow \mathbb{R}$ be a $C^{2}$ function such that $\phi^{\prime \prime} \geq 0$. Suppose that there exists a positive constant $A$ such that

$$
\omega^{1 / 2}\left(t_{1}\right) \omega^{1 / 2}\left(t_{2}\right) \leq\left(\frac{A}{t_{2}-t_{1}} \int_{t_{1}}^{t_{2}} \omega^{3}(t) d t\right)^{1 / 3}
$$

(C) 2014 Choi; licensee Springer. This is an Open Access article distributed under the terms of the Creative Commons Attribution License (http://creativecommons.org/licenses/by/2.0), which permits unrestricted use, distribution, and reproduction in any medium, provided the original work is properly cited. 
holds whenever $t_{1}<t_{2}$ and $\left[t_{1}, t_{2}\right] \subset J$. Let $\mathcal{T}$ be the operator defined as in (1.1). Then there exists a constant $C$ that depends only on $A$ such that

$$
\|\mathcal{T} f\|_{L^{3}\left(\mathbb{R}^{2}\right)} \leq C\|f\|_{L^{3 / 2}\left(\mathbb{R}^{2}\right)}
$$

holds uniformly in $f \in C_{0}^{\infty}\left(\mathbb{R}^{2}\right)$.

Under somewhat stronger assumptions on $\phi(t)$ or $\omega(t)$, the endpoint Lebesgue norm estimate aforementioned can be improved to optimal Lorentz norm estimates, namely from $L^{3 / 2}\left(\mathbb{R}^{2}\right)$ into $L^{3,3 / 2}\left(\mathbb{R}^{2}\right)$ and $L^{3 / 2,3}\left(\mathbb{R}^{2}\right)$ into $L^{3}\left(\mathbb{R}^{2}\right)$. We refer interested readers to [ 6 , $8,10,11]$ for known sufficient conditions for optimal and nearly optimal Lorentz norm estimates. Most importantly, Oberlin established the following uniform optimal Lorentz norm improving estimates.

Theorem 1.2 (Oberlin [11]) Let J be an open interval. Suppose that $\omega(t)$ is monotone increasing and that there exists a positive constant $A$ such that

$$
\sqrt{\omega\left(t_{1}\right) \omega\left(t_{2}\right)} \leq A \omega\left(\left(t_{1}+t_{2}\right) / 2\right)
$$

holds whenever $t_{1}<t_{2}$ and $\left[t_{1}, t_{2}\right] \subset J$. Then the operator $\mathcal{T}$ given by (1.1) satisfies

$$
\begin{aligned}
& \|\mathcal{T} f\|_{L^{3,3 / 2}\left(\mathbb{R}^{2}\right)} \leq C\|f\|_{L^{3 / 2\left(\mathbb{R}^{2}\right)}}, \\
& \|\mathcal{T} f\|_{L^{3}\left(\mathbb{R}^{2}\right)} \leq C\|f\|_{L^{3 / 2,3}\left(\mathbb{R}^{2}\right)}
\end{aligned}
$$

for all $f \in C_{0}^{\infty}\left(\mathbb{R}^{2}\right)$, where $C$ is a constant depending only on $A$.

For the proof of the optimality, see [13] by Stovall along with [8] by Bak et al. It is interesting to ask if the condition in Theorem 1.2 can be relaxed to cover more general curves. Based on an ingenious argument of Oberlin in [11], the author aims to establish a uniform optimal Lorentz norm improving estimate under a condition on averages of the square of $\omega(t)$. The average condition is a slightly stronger version of that in Theorem 1.1, and yet covers most simple plane curves studied up to now including those in Theorem 1.2.

This paper is organized as follows: in the following section, conditions on $\omega(t)$ are introduced and the main theorem is stated. The last section is devoted to the proof of the main theorem. As usual, absolute constants may grow from line to line.

\section{Statement of the main theorem}

Before we state our main result, we introduce certain conditions on functions defined on intervals.

Definition 2.1 Let $0<p<\infty$. For an interval $J_{1}$ in $\mathbb{R}$, a locally $L^{p}$ function $\Phi: J_{1} \rightarrow \mathbb{R}^{+}$, and a positive real number $A$, we let

$$
\begin{aligned}
\mathfrak{G}_{p}(\Phi, A):= & \left\{F: J_{1} \rightarrow \mathbb{R}^{+} \mid \sqrt{F\left(t_{1}\right) F\left(t_{2}\right)} \leq A\left(\frac{1}{t_{2}-t_{1}} \int_{t_{1}}^{t_{2}} \Phi^{p}(t) d t\right)^{1 / p}\right. \\
& \text { whenever } \left.t_{1}<t_{2} \text { and }\left[t_{1}, t_{2}\right] \subset J_{1}\right\}
\end{aligned}
$$


and we let

$$
\mathcal{E}_{p}(A):=\left\{\Phi: J \rightarrow \mathbb{R}^{+} \mid J \text { is an interval and } \Phi \in \mathfrak{G}_{p}(\Phi, A)\right\} .
$$

An interesting subclass of $\mathcal{E}_{p}\left(2^{1 / p} A\right), 0<p<\infty$, was introduced by Bak et al. [14] in studying Fourier restriction estimates related to degenerate curves.

Definition 2.2 For an interval $J$ and a positive real number $A$, a function $\Phi: J \rightarrow \mathbb{R}^{+}$is said to be a member of $\tilde{\mathcal{E}}(A)$ if

- $\Phi$ is monotone; and

- whenever $t_{1}<t_{2}$ and $\left[t_{1}, t_{2}\right] \subset J$,

$$
\sqrt{\Phi\left(t_{1}\right) \Phi\left(t_{2}\right)} \leq A \Phi\left(\left(t_{1}+t_{2}\right) / 2\right)
$$

holds.

The condition (1.2) can be rewritten as $\omega \in \tilde{\mathcal{E}}(A)$.

Remark 2.3 It seems appropriate to mention some properties of $\mathcal{E}_{p}(A)$ and $\tilde{\mathcal{E}}(A)$ mentioned above.

1. It is a simple matter to check:

- $\tilde{\mathcal{E}}(A) \subset \mathcal{E}_{p}\left(2^{1 / p} A\right)$ for all $p \in(0, \infty)$;

- $\Phi \in \mathcal{E}_{p}(A)$ if and only if $\Phi^{p} \in \mathcal{E}_{1}\left(A^{p}\right)$

- $\Phi \in \mathcal{E}_{p}(A)$ implies $\lambda \Phi \in \mathcal{E}_{p}(A)$ for all $\lambda>0$; and

- $\Phi \in \mathcal{E}_{p}(A)$ implies $\Phi(a \cdot+b) \in \mathcal{E}_{p}(A)$ for all $(a, b) \in \mathbb{R} \backslash\{0\} \times \mathbb{R}$.

2. If $0<p_{1}<p_{2}<\infty, \Phi: J \rightarrow \mathbb{R}^{+} \in \mathcal{E}_{p_{1}}(A)$, and $\Phi \in L_{\text {loc }}^{p_{2}}(J)$, then $\Phi \in \mathcal{E}_{p_{2}}(A)$ by Hölder's inequality.

3. The class $\tilde{\mathcal{E}}(1)$ is essentially the class of logarithmically concave functions, which already encompasses many useful examples. Simplest examples are the exponential function and $\Phi(t)=t^{\alpha}, t>0$, for $\alpha \geq 0$. More interesting example is the function $\Phi(t)=e^{-1 / t}, t>0$, which models a curve 'flat' at the origin. A hierarchy of flatter functions that belong to $\tilde{\mathcal{E}}(1)$ was constructed by Bak et al. [14].

4. For a polynomial $p(t)$ of degree $N,|p(t)|$ belongs to $\tilde{\mathcal{E}}\left(2^{N / 2}\right)$ after (possibly) decomposing the real line into at most $3^{N / 2}$ intervals.

5. Nevertheless, there are functions that belong to $\mathcal{E}_{p}(A)$ but do not belong to $\tilde{\mathcal{E}}\left(A^{\prime}\right)$ for any $A^{\prime}>0$. Two examples of curves that our result covers that are not covered in [11] can be constructed with the aid of the examples given below.

Example 2.4 Consider $\Phi_{\beta}(t)=t^{-\beta}, t>0$, for $\beta \geq 2$. Then, for given $0<t_{1}<t_{2}<\infty$, we have by a change of variable

$$
\begin{aligned}
\frac{1}{t_{2}-t_{1}} \int_{t_{1}}^{t_{2}} \Phi_{\beta}(t) d t & =\frac{1}{(\lambda-1) t_{1}^{\beta}} \int_{1}^{\lambda} t^{-\beta} d t \\
& =\frac{1}{(\lambda-1) t_{1}^{\beta}} \int_{\lambda^{-1}}^{1} t^{\beta-2} d t,
\end{aligned}
$$


where $\lambda:=t_{2} / t_{1}>1$. Since $t^{\beta-2}$ is logarithmically concave, we see

$$
\begin{aligned}
\frac{1}{t_{2}-t_{1}} \int_{t_{1}}^{t_{2}} \Phi_{\beta}(t) d t & \geq \frac{1}{2} \frac{1-\lambda^{-1}}{(\lambda-1) t_{1}^{\beta}} \lambda^{(-\beta+2) / 2} \\
& =\frac{1}{2} \frac{1}{t_{1}^{\beta} \lambda^{\beta / 2}}=\frac{1}{2} \sqrt{\Phi_{\beta}\left(t_{1}\right) \Phi_{\beta}\left(t_{2}\right)},
\end{aligned}
$$

which implies $\Phi_{\beta} \in \mathcal{E}_{1}(2)$. In view of Remark 2.3 , given $\beta>0, \Phi_{\beta} \in \mathcal{E}_{p}\left(2^{1 / p}\right)$ if $p \geq 2 / \beta$. One can easily see $\Phi_{\beta} \notin \tilde{\mathcal{E}}\left(A^{\prime}\right)$ for any $A^{\prime}>0$ and $\beta>0$.

Example 2.5 Consider $\Phi:(0, \infty) \rightarrow \mathbb{R}^{+}$given by $\Phi(t)=(2 t)^{1 / 2} e^{t^{2}}$. Then we have $\sqrt{\Phi(t) \Phi(1)} \sim t^{1 / 4} e^{t^{2} / 2}$ and $\Phi((t+1) / 2)=O\left(t^{1 / 2} e^{t^{2} / 3}\right)$ as $t \rightarrow \infty$, which clearly implies $\Phi \notin \tilde{\mathcal{E}}(A)$ for all $A>0$. On the other hand, $\Phi \in \mathcal{E}_{2}(1)$ by the following.

Proposition 2.6 Let $\psi: J \rightarrow \mathbb{R}$. Suppose that $\psi^{\prime} \in \mathcal{E}_{1}(A)$ for some $A>0$. Then the function $\Phi$ given by $\Phi(t)=\left(\psi^{\prime}\right)^{1 / p}(t) \exp (\psi(t))$ belongs to $\mathcal{E}_{p}\left(A^{1 / p}\right)$ for $0<p<\infty$.

Proof Let $t_{1}<t_{2}$. Since $\psi^{\prime} \in \mathcal{E}_{1}(A)$, we have

$$
\psi\left(t_{2}\right)-\psi\left(t_{1}\right)=\int_{t_{1}}^{t_{2}} \psi^{\prime}(t) d t \geq A^{-1}\left(t_{2}-t_{1}\right) \sqrt{\psi^{\prime}\left(t_{1}\right) \psi^{\prime}\left(t_{2}\right)}>0
$$

by the fundamental theorem of calculus and the assumption on $\psi^{\prime}(t)$. A change of variable gives

$$
\begin{aligned}
\frac{1}{t_{2}-t_{1}} \int_{t_{1}}^{t_{2}} \Phi^{p}(t) d t & =\frac{1}{t_{2}-t_{1}} \int_{t_{1}}^{t_{2}} e^{p \psi(t)} \psi^{\prime}(t) d t \\
& =\frac{1}{p\left(t_{2}-t_{1}\right)} \int_{p \psi\left(t_{1}\right)}^{p \psi\left(t_{2}\right)} e^{t} d t \\
& =\frac{\psi\left(t_{2}\right)-\psi\left(t_{1}\right)}{t_{2}-t_{1}} \times \frac{e^{p \psi\left(t_{2}\right)}-e^{p \psi\left(t_{1}\right)}}{p\left(\psi\left(t_{2}\right)-\psi\left(t_{1}\right)\right)}
\end{aligned}
$$

From

$$
\begin{aligned}
\frac{e^{b}-e^{a}}{b-a} & =e^{(b+a) / 2} \times \frac{e^{(b-a) / 2}-e^{-(b-a) / 2}}{2 \times(b-a) / 2} \\
& =e^{(b+a) / 2} \times \frac{\sinh ((b-a) / 2)}{(b-a) / 2} \geq e^{(b+a) / 2}
\end{aligned}
$$

for all $a<b$, we see

$$
\frac{e^{p \psi\left(t_{2}\right)}-e^{p \psi\left(t_{1}\right)}}{p\left(\psi\left(t_{2}\right)-\psi\left(t_{1}\right)\right)} \geq e^{p\left(\psi\left(t_{1}\right)+\psi\left(t_{2}\right)\right) / 2}
$$

Altogether, we obtain

$$
\frac{1}{t_{2}-t_{1}} \int_{t_{1}}^{t_{2}} \Phi^{p}(t) d t \geq A^{-1} e^{p\left(\psi\left(t_{1}\right)+\psi\left(t_{2}\right)\right) / 2} \sqrt{\psi^{\prime}\left(t_{1}\right) \psi^{\prime}\left(t_{2}\right)}=A^{-1}\left(\Phi\left(t_{1}\right) \Phi\left(t_{2}\right)\right)^{p / 2} .
$$

By taking the $p$ th root we obtain the desired estimate. 
We are now ready to state the main theorem of this paper.

Theorem 2.7 Let $-\infty \leq a<b \leq \infty$, and let $\phi:(a, b) \rightarrow \mathbb{R}$ be a $C^{2}$ function such that $\phi^{\prime \prime} \geq 0$ on the interval. Suppose that there exists a positive constant $A$ such that $\omega \in \mathcal{E}_{2}(A)$, i.e.

$$
\omega^{1 / 2}\left(t_{1}\right) \omega^{1 / 2}\left(t_{2}\right) \leq A\left(\frac{1}{t_{2}-t_{1}} \int_{t_{1}}^{t_{2}} \omega^{2}(t) d t\right)^{1 / 2}
$$

holds whenever $a<t_{1}<t_{2}<b$. Let $\mathcal{T}$ be the operator given by (1.1). Then there exists $a$ constant $C$ that depends only on $A$ such that

$$
\begin{aligned}
& \|\mathcal{T} f\|_{L^{3,3 / 2}\left(\mathbb{R}^{2}\right)} \leq C\|f\|_{L^{3 / 2\left(\mathbb{R}^{2}\right)}}, \\
& \|\mathcal{T} f\|_{L^{3}\left(\mathbb{R}^{2}\right)} \leq C\|f\|_{L^{3 / 2,3}\left(\mathbb{R}^{2}\right)}
\end{aligned}
$$

holds uniformly in $f \in C_{0}^{\infty}\left(\mathbb{R}^{2}\right)$.

Remark 2.8 Some remarks are in order.

- In view of Remark 2.3, Proposition 2.6, Example 2.4 and Example 2.5, the condition $\omega \in \tilde{\mathcal{E}}(A)$ is strictly stronger than the condition $\omega \in \mathcal{E}_{2}(\sqrt{2} A)$ in Theorem 2.7, and therefore our result improves Theorem 1.2.

- An explicit example is also available. Consider $\phi(t)=t^{-1 / 2} \exp \left(t^{2}\right)$ defined for $t \in(c, \infty)$, where $c$ is a large constant. A simple calculation shows $\omega(t) \sim t^{1 / 2} \exp \left(t^{2} / 3\right)$. By Proposition 2.6, $\omega \in \mathcal{E}_{2}(A)$ for some $A>0$. Thus, the corresponding operator $\mathcal{T}$ satisfies endpoint Lorentz estimates (2.1) and (2.2) by Theorem 2.7, but Theorem 1.2 is not directly applicable.

- It is not known whether $\omega \in \mathcal{E}_{2}(A)$ in Theorem 2.7 can be further relaxed to $\omega \in \mathcal{E}_{p}(A)$ for some $p>2$. More generally, one can ask for the optimal $p$ such that $\omega \in \mathcal{E}_{p}(A)$ guarantees the boundedness of $\mathcal{T}$ from $L^{\frac{3}{2}, q}\left(\mathbb{R}^{2}\right)$ to $L^{3, r}\left(\mathbb{R}^{2}\right)$ for given $q \leq r$.

\section{Proof of the main theorem}

Before we prove the theorem, we begin with a couple of lemmas.

Lemma 3.1 Let $J$ be an interval in $\mathbb{R}$, and let $\omega: J \rightarrow \mathbb{R}_{+}$be a continuous function such that $\omega \in \mathcal{E}_{2}(A)$ for some $A>0$, i.e.

$$
\omega^{1 / 2}\left(t_{1}\right) \omega^{1 / 2}\left(t_{2}\right) \leq A\left(\frac{1}{t_{2}-t_{1}} \int_{t_{1}}^{t_{2}} \omega^{2}(t) d t\right)^{1 / 2}
$$

holds whenever $t_{1}<t_{2}$ and $\left[t_{1}, t_{2}\right] \subset J$. Then the following holds:

$$
\omega\left(t_{1}\right)^{1 / 3} \omega\left(t_{2}\right)^{1 / 3} \omega\left(t^{*}\right)^{1 / 3} \leq 6^{1 / 3} A\left(\frac{1}{t_{2}-t_{1}} \int_{t_{1}}^{t_{2}} \omega^{3}(t) d t\right)^{1 / 3}
$$

whenever $t_{1}<t_{2}$ and $t^{*} \in\left[t_{1}, t_{2}\right] \subset J$.

Proof of Lemma 3.1 Let $t^{*} \in\left[t_{1}, t_{2}\right] \subset J$. From

$$
\omega^{1 / 2}\left(t_{1}\right) \omega^{1 / 2}\left(t_{2}\right) \leq A\left(\frac{1}{t_{2}-t_{1}} \int_{t_{1}}^{t_{2}} \omega^{2}(t) d t\right)^{1 / 2},
$$


we obtain

$$
\begin{aligned}
& \omega^{1 / 2}\left(t_{1}\right) \omega^{1 / 2}\left(t_{2}\right) \omega^{1 / 2}\left(t^{*}\right) \\
& \leq A\left(\frac{1}{t_{2}-t_{1}} \int_{t_{1}}^{t_{2}} \omega^{2}(t) \omega\left(t^{*}\right) d t\right)^{1 / 2} \\
& \leq A^{3 / 2}\left(\frac{1}{t_{2}-t_{1}} \int_{t_{1}}^{t_{2}} \omega(t)\left|\frac{1}{t^{*}-t} \int_{t}^{t^{*}} \omega^{2}(s) d s\right| d t\right)^{1 / 2} \\
& \leq A^{3 / 2}\left(\frac{1}{t_{2}-t_{1}} \int_{t_{1}}^{t_{2}} \omega^{3}(t) d t\right)^{1 / 6} \\
& \quad \times\left(\frac{1}{t_{2}-t_{1}} \int_{t_{1}}^{t_{2}}\left|\frac{1}{t^{*}-t} \int_{t}^{t^{*}} \omega^{2}(s) d s\right|^{3 / 2} d t\right)^{1 / 3}
\end{aligned}
$$

by hypothesis and Hölder's inequality. Applying Hardy's inequality twice gives us

$$
\left(\int_{t_{1}}^{t_{2}}\left|\frac{1}{t^{*}-t} \int_{t}^{t^{*}} \omega^{2}(s) d s\right|^{3 / 2} d t\right)^{2 / 3} \leq 6\left(\int_{t_{1}}^{t_{2}} \omega^{3}(t) d t\right)^{2 / 3}
$$

and so we obtain

$$
\omega^{1 / 2}\left(t_{1}\right) \omega^{1 / 2}\left(t_{2}\right) \omega^{1 / 2}\left(t^{*}\right) \leq \frac{6^{1 / 2} A^{3 / 2}}{\left(t_{2}-t_{1}\right)^{1 / 2}}\left(\int_{t_{1}}^{t_{2}} \omega^{3}(t) d t\right)^{1 / 2} .
$$

By taking the 2/3th power, we obtain the desired estimate.

The following lemma, which is nearly a triviality, generalizes a version of Lemma 2.2 in [11].

Lemma 3.2 Suppose $F$ is nonnegative and continuous on some interval $[a, b]$. For $t \in[a, b]$, we let $\tilde{F}(t):=\max _{[t, b]} F$, and for $\rho>0$, we let

$$
E_{\rho}=\{t \in[a, b]: \tilde{F}(t)(b-t) \leq \rho\} .
$$

Then we have

$$
\int_{E_{\rho}} F(t) d t \leq \rho
$$

Proof of Lemma 3.2 Observe that the function $t \mapsto \tilde{F}(t)(b-t)$ is a monotone decreasing function. Let $\rho>0$ be given. Since $b \in E_{\rho}, E_{\rho}$ is nonempty. Let $t_{*}:=\inf E_{\rho}$. Then we have $\tilde{F}\left(t_{*}\right)\left(b-t_{*}\right) \leq \rho$. From this, we obtain

$$
\begin{aligned}
\int_{E_{\rho}} F(t) d t & =\int_{t_{*}}^{b} F(t) d t \\
& \leq \tilde{F}\left(t_{*}\right)\left(b-t_{*}\right)=\rho,
\end{aligned}
$$

which finishes the proof. 
Proof of Theorem 2.7 It suffices to prove (2.1) by duality. We may further assume, without loss of generality, $-\infty<a<b<\infty$, since a uniform estimate independent of $a$ and $b$ will allow us a suitable limiting argument. For a measurable subset $E$ of either $\mathbb{R}$ or $\mathbb{R}^{2}$, we denote the Lebesgue measure and the characteristic function of $E$ by $|E|$ and $\mathbb{1}_{E}$, respectively. We also write $\gamma(t)=(t, \phi(t))$.

By a well-known interpolation argument $[7,8]$, it suffices to show that

$$
\int_{a}^{b}\left(\int_{a}^{b} \mathbb{1}_{E}\left(\gamma\left(t_{2}\right)-\gamma\left(t_{1}\right)\right) \omega\left(t_{1}\right) d t_{1}\right)^{2} \omega\left(t_{2}\right) d t_{2} \leq C|E|
$$

holds for measurable sets $E \subset \mathbb{R}^{2}$. In view of the simple identities

$$
\begin{aligned}
& \int_{a}^{b}\left(\int_{t_{2}}^{b} \mathbb{1}_{E}\left(\gamma\left(t_{2}\right)-\gamma\left(t_{1}\right)\right) \omega\left(t_{1}\right) d t_{1}\right)^{2} \omega\left(t_{2}\right) d t_{2} \\
& \quad=\int_{a}^{b}\left(\int_{a}^{a+b-t_{2}} \mathbb{1}_{E}\left(\gamma\left(t_{2}\right)-\gamma\left(a+b-t_{1}\right)\right) \omega\left(a+b-t_{1}\right) d t_{1}\right)^{2} \omega\left(t_{2}\right) d t_{2} \\
& =\int_{a}^{b}\left(\int_{a}^{t_{2}} \mathbb{1}_{E}\left(\gamma\left(a+b-t_{2}\right)-\gamma\left(a+b-t_{1}\right)\right) \omega\left(a+b-t_{1}\right) d t_{1}\right)^{2} \omega\left(a+b-t_{2}\right) d t_{2} \\
& =\int_{a}^{b}\left(\int_{a}^{t_{2}} \mathbb{1}_{E}\left(\bar{\gamma}\left(t_{2}\right)-\bar{\gamma}\left(t_{1}\right)\right) \bar{\omega}\left(t_{1}\right) d t_{1}\right)^{2} \bar{\omega}\left(t_{2}\right) d t_{2},
\end{aligned}
$$

where $\bar{\gamma}(t):=(t, \bar{\phi}(t)), \bar{\phi}(t):=\phi(a+b-t), \bar{\omega}(t):=\left(\bar{\phi}^{\prime \prime}(t)\right)^{1 / 3}=\omega(a+b-t) \in \mathcal{E}_{2}(A)$, and $\bar{E}:=\left\{\left(x_{1}, x_{2}\right):\left(-x_{1}, x_{2}\right) \in E\right\}$, it is enough to establish that

$$
\int_{a}^{b}\left(\int_{a}^{t_{2}} \mathbb{1}_{E}\left(\gamma\left(t_{2}\right)-\gamma\left(t_{1}\right)\right) \omega\left(t_{1}\right) d s_{t}\right)^{2} \omega\left(t_{2}\right) d t_{2} \leq C|E|
$$

holds for measurable sets $E \subset \mathbb{R}^{2}$. To do this, we let $\Delta:=\left\{\left(t_{1}, t_{2}\right): a<t_{1}<t_{2}<b\right\}$. The mapping $\Phi: \Delta \rightarrow \mathbb{R}^{2}$ given by $\Phi\left(t_{1}, t_{2}\right)=\gamma\left(t_{2}\right)-\gamma\left(t_{1}\right)$ is one-to-one and the absolute value of the Jacobian determinant $J\left(t_{1}, t_{2}\right)$ of $\Phi$ is given by

$$
J\left(t_{1}, t_{2}\right)=\phi^{\prime}\left(t_{2}\right)-\phi^{\prime}\left(t_{1}\right)
$$

Given measurable $\Omega \subset \Delta$ and $t_{2} \in(a, b)$, we apply Lemma 3.2 with

$$
\rho=\frac{1}{2} \int_{a}^{t_{2}} \mathbb{1}_{\Omega}\left(t_{1}, t_{2}\right) \omega\left(t_{1}\right) d t_{1}
$$

to obtain

$$
\int_{\tilde{\omega}\left(t_{1} ; t_{2}\right)\left(t_{2}-t_{1}\right) \leq \rho} \mathbb{1}_{\Omega}\left(t_{1}, t_{2}\right) \omega\left(t_{1}\right) d t_{1} \leq \frac{1}{2} \int_{a}^{t_{2}} \mathbb{1}_{\Omega}\left(t_{1}, t_{2}\right) \omega\left(t_{1}\right) d t_{1},
$$

where $\tilde{\omega}\left(t_{1} ; t_{2}\right):=\max _{\left[t_{1}, t_{2}\right]} \omega$. From this, we get

$$
\int_{\tilde{\omega}\left(t_{1} ; t_{2}\right)\left(t_{2}-t_{1}\right) \geq \rho} \mathbb{1}_{\Omega}\left(t_{1}, t_{2}\right) \omega\left(t_{1}\right) d t_{1} \geq \frac{1}{2} \int_{a}^{t_{2}} \mathbb{1}_{\Omega}\left(t_{1}, t_{2}\right) \omega\left(t_{1}\right) d t_{1},
$$


and so

$$
\begin{aligned}
\frac{1}{4}\left(\int_{a}^{t_{2}} \mathbb{1}_{\Omega}\left(t_{1}, t_{2}\right) \omega\left(t_{1}\right) d t_{1}\right)^{2} & \leq \rho \int_{\tilde{\omega}\left(t_{1} ; t_{2}\right)\left(t_{2}-t_{1}\right) \geq \rho} \mathbb{1}_{\Omega}\left(t_{1}, t_{2}\right) \omega\left(t_{1}\right) d t_{1} \\
& \leq \int_{\tilde{\omega}\left(t_{1} ; t_{2}\right)\left(t_{2}-t_{1}\right) \geq \rho} \mathbb{1}_{\Omega}\left(t_{1}, t_{2}\right) \omega\left(t_{1}\right) \tilde{\omega}\left(t_{1} ; t_{2}\right)\left(t_{2}-t_{1}\right) d t_{1} \\
& \leq \int_{a}^{t_{2}} \mathbb{1}_{\Omega}\left(t_{1}, t_{2}\right) \omega\left(t_{1}\right) \tilde{\omega}\left(t_{1} ; t_{2}\right)\left(t_{2}-t_{1}\right) d t_{1} .
\end{aligned}
$$

Multiplying by $\omega\left(t_{2}\right)$ and integrating with respect to $t_{2}$ provides us with

$$
\begin{aligned}
& \int_{a}^{b}\left(\int_{a}^{t_{2}} \mathbb{1}_{\Omega}\left(t_{1}, t_{2}\right) \omega\left(t_{1}\right) d t_{1}\right)^{2} \omega\left(t_{2}\right) d t_{2} \\
& \quad \leq 4 \int_{a}^{b} \int_{a}^{t_{2}} \mathbb{1}_{\Omega}\left(t_{1}, t_{2}\right) \omega\left(t_{1}\right) \omega\left(t_{2}\right) \tilde{\omega}\left(t_{1} ; t_{2}\right)\left(t_{2}-t_{1}\right) d t_{1} d t_{2} .
\end{aligned}
$$

Notice that for $a<t_{1}<t_{2}<b$, there exists $t_{*} \in\left[t_{1}, t_{2}\right]$ such that $\tilde{\omega}\left(t_{1} ; t_{2}\right)=\omega\left(t_{*}\right)$. By Lemma 3.1, we have

$$
\begin{aligned}
\omega\left(t_{1}\right) \omega\left(t_{2}\right) \tilde{\omega}\left(t_{1} ; t_{2}\right)\left(t_{2}-t_{1}\right) & =\omega\left(t_{1}\right) \omega\left(t_{2}\right) \omega\left(t_{*}\right)\left(t_{2}-t_{1}\right) \\
& \leq 6 A^{3} \int_{t_{1}}^{t_{2}} \omega^{3}(t) d t \\
& =6 A^{3} \int_{t_{1}}^{t_{2}} \phi^{\prime \prime}(t) d t \\
& =6 A^{3}\left(\phi^{\prime}\left(t_{2}\right)-\phi^{\prime}\left(t_{1}\right)\right) \\
& =6 A^{3} J\left(t_{1}, t_{2}\right),
\end{aligned}
$$

which further implies

$$
\int_{a}^{b}\left(\int_{a}^{t_{2}} \mathbb{1}_{\Omega}\left(t_{1}, t_{2}\right) \omega\left(t_{1}\right) d t_{1}\right)^{2} \omega\left(t_{2}\right) d t_{2} \leq 24 A^{3} \int_{a}^{b} \int_{a}^{b} \mathbb{1}_{\Omega}\left(t_{1}, t_{2}\right) J\left(t_{1}, t_{2}\right) d t_{2} d t_{1}
$$

Letting $\Omega=\left\{\left(t_{1}, t_{2}\right) \in \Delta: \gamma\left(t_{1}\right)-\gamma\left(t_{2}\right) \in E\right\}$ and making a change of variables, we obtain the desired estimate (3.2).

\section{Competing interests}

The author declares that he has no competing interests.

\section{Acknowledgements}

The author is grateful to the anonymous referee for valuable comments. This paper was completed with Ajou University research fellowship of 2013

Received: 8 January 2014 Accepted: 1 July 2014 Published: 22 July 2014

\section{References}

1. Drury, SW, Marshall, BP: Fourier restriction theorems for curves with affine and Euclidean arclengths. Math. Proc. Camb. Philos. Soc. 97(1), 111-125 (1985)

2. Guggenheimer, HW: Differential Geometry. McGraw-Hill, New York (1963)

3. Drury, SW: Degenerate curves and harmonic analysis. Math. Proc. Camb. Philos. Soc. 108(1), 89-96 (1990)

4. Oberlin, DM: Affine dimension: measuring the vestiges of curvature. Mich. Math. J. 51(1), 13-26 (2003) 
5. Choi, Y: Convolution operators with the affine arclength measure on plane curves. J. Korean Math. Soc. 36(1), 193-207 (1999)

6. Oberlin, DM: Convolution with measures on hypersurfaces. Math. Proc. Camb. Philos. Soc. 129(3), $517-526$ (2000)

7. Oberlin, DM: Two estimates for curves in the plane. Proc. Am. Math. Soc. 132(11), 3195-3201 (2004)

8. Bak, J-G, Oberlin, DM, Seeger, A: Two end point bounds for generalized Radon transforms in the plane. Rev. Mat. Iberoam. 18(1), 231-247 (2002)

9. Oberlin, DM: Convolution with measures on polynomial curves. Math. Scand. 90(1), 126-138 (2002)

10. Dendrinos, S, Laghi, N, Wright, J: Universal $L^{p}$ improving for averages along polynomial curves in low dimensions. J. Funct. Anal. 257(5), 1355-1378 (2009)

11. Oberlin, DM: Convolution with measures on flat curves in low dimensions. J. Funct. Anal. 259(7), 1799-1815 (2010)

12. Choi, Y: Convolution estimates related to space curves. J. Inequal. Appl. 2011, 91 (2011)

13. Stovall, B: Endpoint bounds for a generalized Radon transform. J. Lond. Math. Soc. 80(2), 357-374 (2009)

14. Bak, J-G, Oberlin, DM, Seeger, A: Restriction of Fourier transforms to curves. II. Some classes with vanishing torsion. J. Aust. Math. Soc. 85(1), 1-28 (2008)

doi:10.1186/1029-242X-2014-256

Cite this article as: Choi: Uniform Lorentz norm estimates for convolution operators. Journal of Inequalities and Applications 2014 2014:256.

\section{Submit your manuscript to a SpringerOpen ${ }^{\ominus}$ journal and benefit from:}

- Convenient online submission

- Rigorous peer review

- Immediate publication on acceptance

- Open access: articles freely available online

- High visibility within the field

- Retaining the copyright to your article 